\title{
UNA MIRADA A LOS INSECTOS ACUÁTICOS DE LA COLECCIÓN DEL DEPARTAMENTO DE BIOLOGÍA DELA UNIVERSIDAD PEDAGÓGICA NACIONALDE COLOMBIA, SEDE BOGOTÁ
}

\section{A Glance at Aquatic Insects from the Collection of the Department of Biology from Universidad Pedagógica Nacional}

\section{Andrea Castiblanco Zerda ${ }^{1}$}

Fecha de recepción: 5 de abril de 2016

Fecha de aprobación: 9 de mayo de 2016

La colección de insectos acuáticos de la Licenciatura en Biología ha procurado mantener un compendio de especímenes de los diferentes órdenes de insectos acuáticos y macroinvertebrados, que registra la diversidad de zonas del país. Esto se ha llevado a cabo a través del ejercicio de colecta realizado en las diferentes salidas de campo que propone el departamento, enfocado, desde luego, en la enseñanza y reconocimiento de las especies presentes en medios acuáticos.

Por ello, esta pequeña muestra de insectos acuáticos con los que cuenta la colección da evidencia del arduo trabajo que por años ha llevado a cabo en la formación de licenciados en Biología. Muestra la importancia de los compendios biológicos como una de las vías para la producción de trabajos de aula, laboratorios e investigaciones, que permiten que el maestro en formación tenga herramientas para el desarrollo de habilidades de manejo de muestras, observación de especímenes y técnicas de uso de los diferentes materiales que la colección puede aportar.

1 Licenciada en Biología, Universidad Pedagógica Nacional de Colombia, sede Bogotá. 2016. Correo electrónico: andreacastiblanoz@gmail. com 


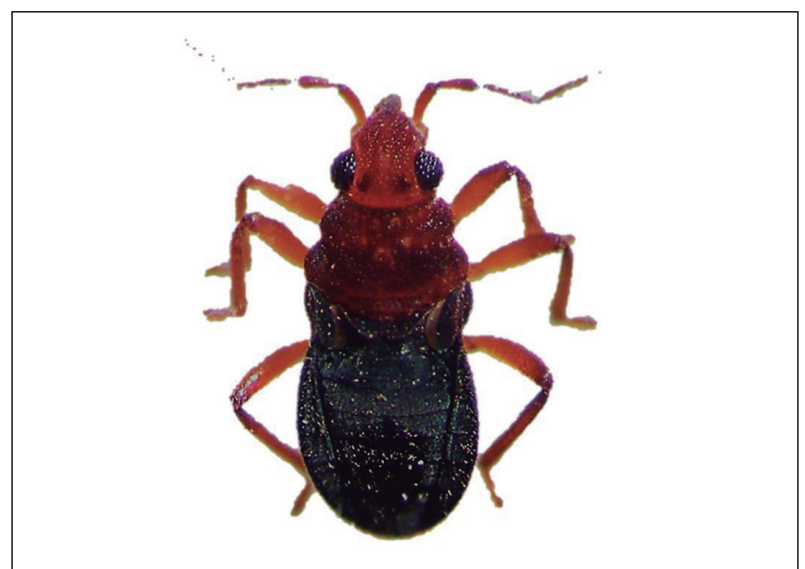

Orden: Hemiptera

Familia: Hebridae

Fotografía tomada por Castiblanco-Zerda, A. y Pinzón-Osorio, C.

Estos organismos son indicadores de aguas oligomesotróficas y se encuentran en sustratos rocosos, arenosos o en plantas sumergidas (Roldán, 1988). Este orden en especial tiene dentro de su diversidad variedad de morfoespecies que son ampliamente distribuidos en ambientes acuáticos lénticos. Este grupo puede ser el predilecto para mostrar las diferentes adaptaciones para sobrevivir en ambientes acuáticos.

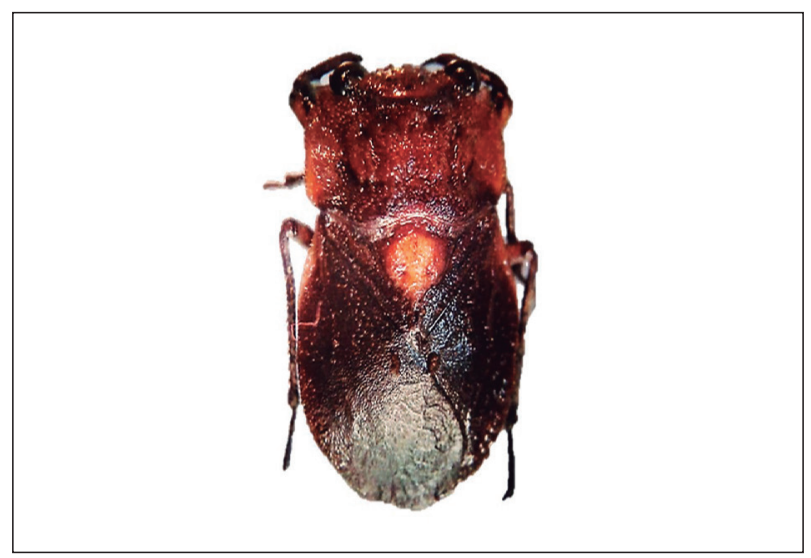

Orden: Hemiptera

Familia: Gelastocoridae

Fotografía tomada por Castiblanco-Zerda, $A$.

Presentes en cuerpos de agua lóticos (Domínguez \& Fernández, 2009). Indican ambientes mesoeutróficos, estos organismos poseen patas delanteras que les permite agarrar fuertemente a su presa o sostenerse de la vegetación acuática. El aspecto de esta familia es particular gracias a la posición de sus ojos y sus robustas patas anteriores. Esta familia permite ejemplificar el aspecto depredador de los insectos acuáticos.

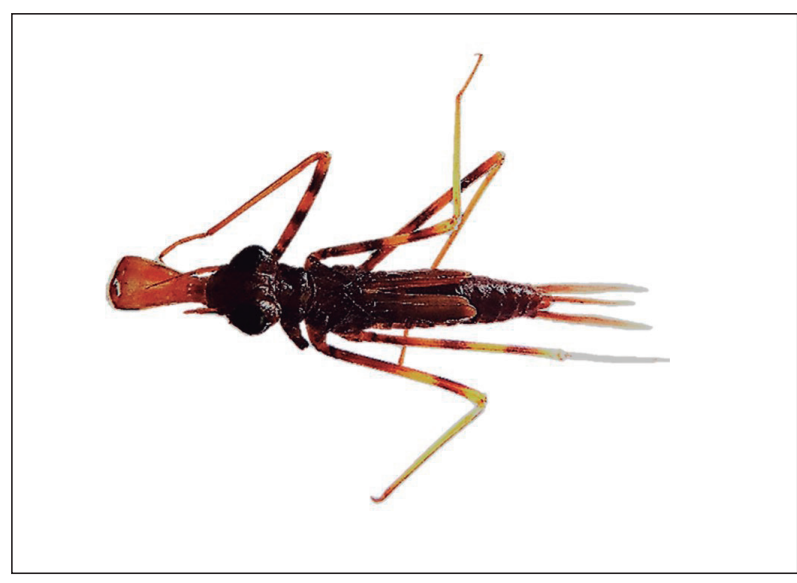

Orden: Odonata

Familia: Megapodagrionidae

Fotografía tomada por Castiblanco-Zerda, $A$.

Se encuentran en ecosistemas acuáticos lóticos, se caracterizan por habitar en aguas limpias con buenas condiciones fisicoquímicas (Roldán, 1988). Los odonatos permiten evidenciar la adaptabilidad de los insectos en ambientes tanto acuáticos, riparios y terrestres. Son organismos útiles en temáticas de adaptación, metamorfosis y funciones ecosistémicas debido a que, siendo un grupo depredador, controlan poblaciones de otros insectos, con preferencia en dípteros.

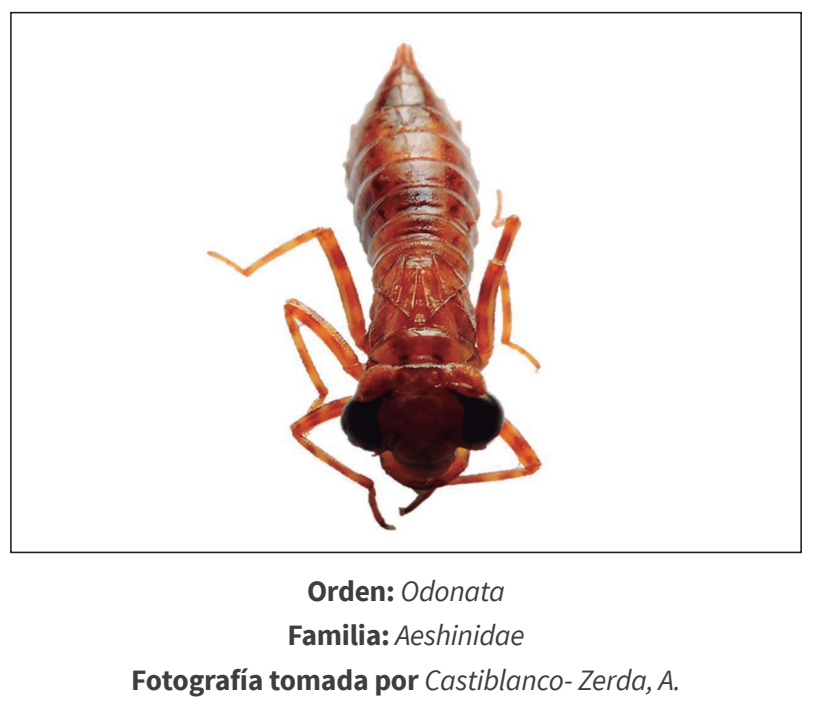

Estos organismos gustan de la abundante vegetación o sustratos lodosos, cumplen una función bioindicadora de aguas mesotróficas (Roldán, 1988). Son capaces de capturar alevinos de peces.

Los insectos acuáticos tienen diferentes funciones ecosistémicas, ya sean depredadores, detritívoros, herbívoros o descomponedores, lo cual es un claro ejemplo de la 
importancia para los ecosistemas en donde sobreviven, por ello, son actores predilectos para la enseñanza de la ecología y la caracterización de los sistemas acuáticos.

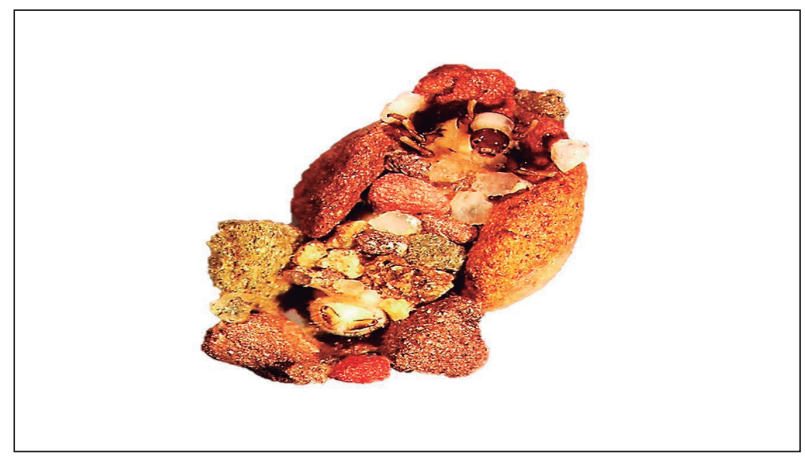

Orden: Trichoptera

Familia: Glossomatidae

Fotografía tomada por Castiblanco-Zerda, A.

Este orden se caracteriza porque la mayoría de sus familias construyen casas con una seda o líquido que secretan, lo cual es útil para proteger sus cuerpos blandos o filtrar su comida por las estructuras que crean utilizando materia vegetal o mineral.

Esta familia en especial filtra el sustrato raspándolo de donde se encuentra adherido.

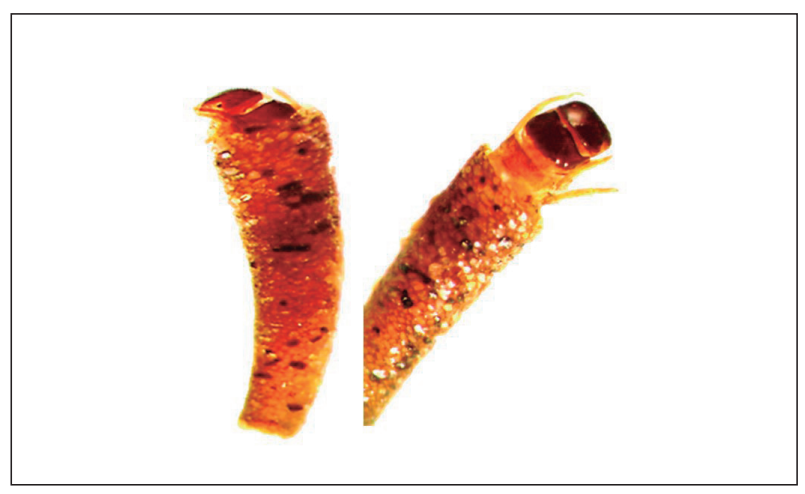

Orden: Trichoptera

Familia: Anomalopsychidae

Fotografía tomada por Castiblanco-Zerda, $A$.

Insectos que elaboran su casa con arena fina dándole forma de cono. Estos organismos gustan de corrientes medianamente fuertes y son perfectos para los ejercicios de colecta, debido a su forma característica que es atractiva y fácilmente identificable. Por otro lado, estos organismos son bioindicadores, permitiendo hacer evaluación de la calidad del agua.

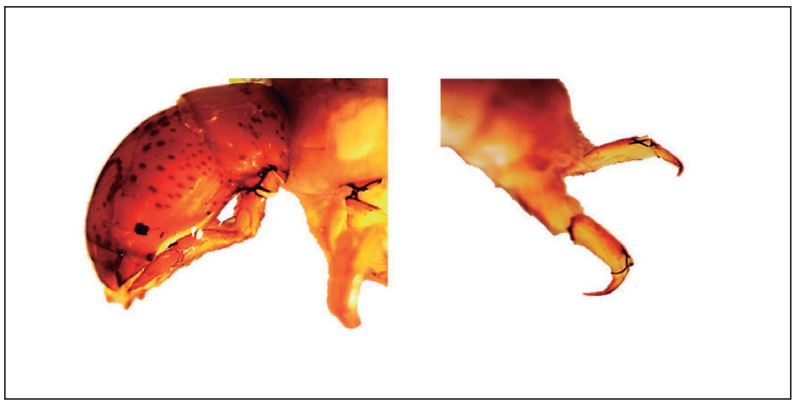

Orden: Trichoptera

Familia: Polycentropodidae

Fotografía tomada por Castiblanco-Zerda, A. y Pinzón-Osorio, C.

La colección de insectos acuáticos del Departamento de Biología tiene un enfoque especial en el orden Trichoptera, el cual se encuentra con estándares de clasificación avanzados. Esto permite a los estudiantes interesados el adelanto de trabajos prácticos y el aprovechamiento de los especímenes en proyectos por desarrollar; a la hora de implementarlos, constituyen una ventaja en las bases de datos que posee la colección.

Actualmente, se han iniciado trabajos de grado y pasantías que permitirán a los demás órdenes de insectos acuáticos tener dichas bases de datos actualizadas. Así, los licenciados en formación podrán desarrollar estudios con estos materiales biológicos, aprovechando aún más los recursos del departamento.

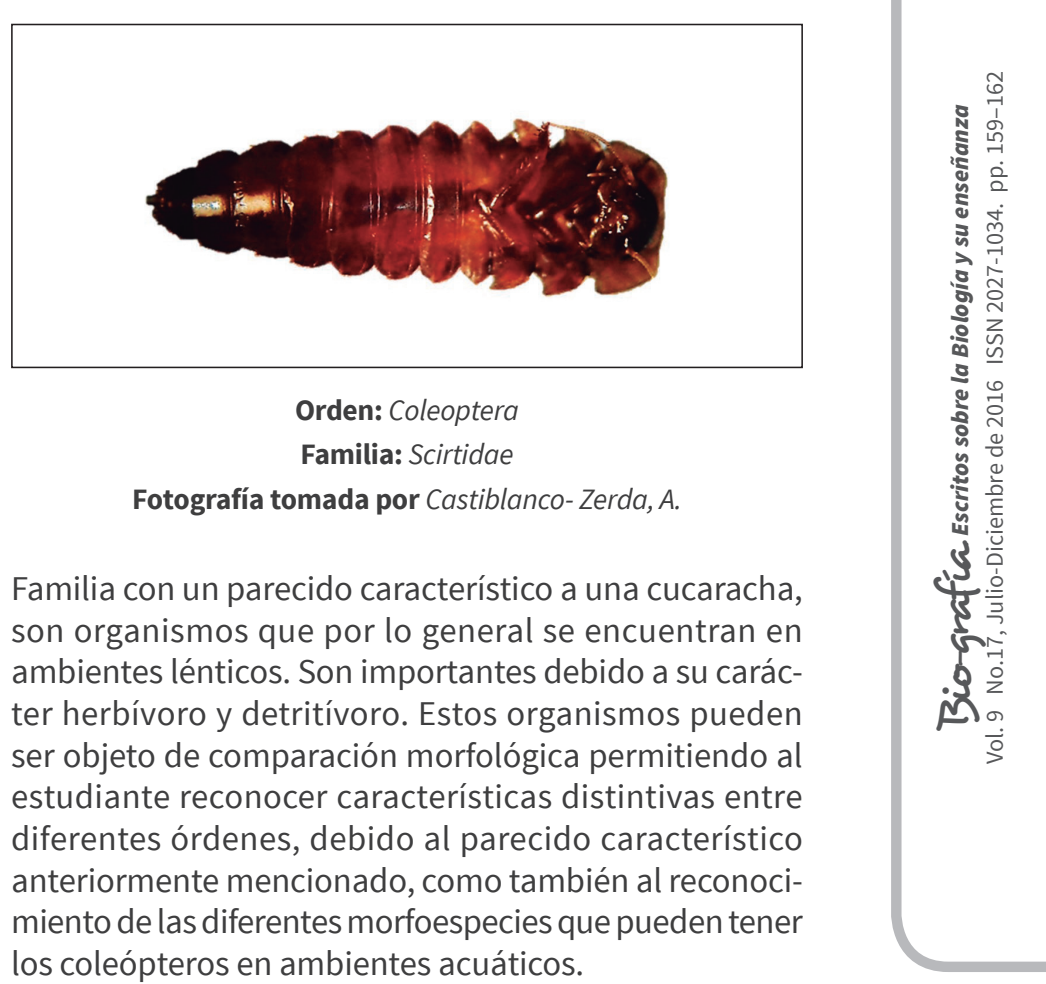




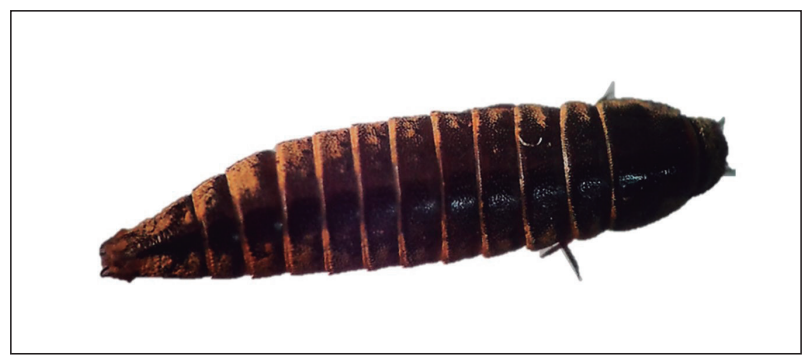

Orden: Coleoptera

Familia: Elmidae

Fotografía tomada por Castiblanco-Zerda, $A$.

Esta es una morfoespecie que representa a los elmidos, claramente es uno de los grupos que mayor información ecológica ofrece a diferencia de otros grupos o familias del mismo orden.

La importancia de estudiar los insectos radica en el amplio conocimiento que pueden rendir en la conservación y evaluación de la calidad de las aguas de los diferentes ríos, quebradas o lagos, siendo estos fundamentales para la enseñanza de temáticas biológicas como medio próximo, en un país en donde los recursos hídricos saltan a la vista. Es por ello que, los compendios biológicos como las colecciones alcohólicas de la Universidad Pedagógica Nacional de Colombia, permiten un acercamiento a estos ambientes y aportan al reconocimiento de la diversidad que las aguas colombianas ocultan.

\section{Agradecimientos}

Esta toma fotográfica no se hubiera realizado sin la autorización del profesor Francisco Medellín, quien muy amablemente la permitió, y sin el fotógrafo Cesar Augusto Pinzón Osorio, quien trabajó conjuntamente en el proceso.

\section{Referencias}

Domínguez, E. \& Fernández, H. R. (2009). Macroinvertebrados bentónicos sudamericanos. Sistemática y biología. Tucumán, Argentina: Fundación Miguel Lillo.

Roldán, G. (1988). Guía para el estudio de los macroinvertebrados acuáticos del Departamento de Antioquia. Universidad de Antioquia. Centro de investigaciones CIEN. Bogotá: Presencia. 